\title{
Improved measurements of the absolute branching fractions of the inclusive decays $D^{+(0)} \rightarrow \phi X$
}

M. Ablikim, ${ }^{1}$ M. N. Achasov, ${ }^{10, d}$ P. Adlarson ${ }^{59}$ S. Ahmed,${ }^{15}$ M. Albrecht,${ }^{4}$ M. Alekseev,${ }^{58 a, 58 c}$ A. Amoroso, ${ }^{58 a, 58 c}$ F. F. An,${ }^{1}$ Q. An ${ }^{55,43}$ Y. Bai, ${ }^{42}$ O. Bakina, ${ }^{27}$ R. Baldini Ferroli, ${ }^{23 a}$ I. Balossino, ${ }^{24 a}$ Y. Ban, ${ }^{35}$ K. Begzsuren, ${ }^{25}$ J. V. Bennett, ${ }^{5}$ N. Berger ${ }^{26}$ M. Bertani, ${ }^{23 a}$ D. Bettoni, ${ }^{24 a}$ F. Bianchi, ${ }^{58 a}, 58 \mathrm{c}$ J. Biernat, ${ }^{59}$ J. Bloms, ${ }^{52}$ I. Boyko, ${ }^{27}$ R. A. Briere, ${ }^{5}$ H. Cai, ${ }^{60}$ X. Cai, ${ }^{1,43}$ A. Calcaterra, ${ }^{23 a}$ G. F. Cao ${ }^{1,47}$ N. Cao, ${ }^{1,47}$ S. A. Cetin, ${ }^{46 b}$ J. Chai,${ }^{58 c}$ J. F. Chang, ${ }^{1,43}$ W. L. Chang, ${ }^{1,47}$ G. Chelkov, ${ }^{27, b, c}$ D. Y. Chen, ${ }^{6}$ G. Chen, ${ }^{1}$ H. S. Chen, ${ }^{1,47}$ J. C. Chen, ${ }^{1}$ M. L. Chen, ${ }^{1,43}$ S. J. Chen, ${ }^{33}$ Y. B. Chen, ${ }^{1,43}$ W. Cheng, ${ }^{58 c}$ G. Cibinetto ${ }^{24 a}$ F. Cossio, ${ }^{58 c}$ X. F. Cui,${ }^{34}$ H. L. Dai ${ }^{1,43}$ J. P. Dai ${ }^{38, h}$ X. C. Dai, ${ }^{1,47}$ A. Dbeyssi, ${ }^{15}$ D. Dedovich,${ }^{27}$ Z. Y. Deng, ${ }^{1}$ A. Denig, ${ }^{26}$ I. Denysenko, ${ }^{27}$ M. Destefanis, ${ }^{58 a, 58 c}$ F. De Mori ${ }^{58 a, 58 c}$ Y. Ding, ${ }^{31}$ C. Dong, ${ }^{34}$ J. Dong,,${ }^{1,43}$ L. Y. Dong, ${ }^{1,47}$ M. Y. Dong, ${ }^{1,43,47}$ Z. L. Dou, ${ }^{33}$ S. X. Du, ${ }^{63}$ J. Z. Fan, ${ }^{45}$ J. Fang, ${ }^{1,43}$ S. S. Fang, ${ }^{1,47}$ Y. Fang, ${ }^{1}$ R. Farinelli, ${ }^{24 a, 24 b}$ L. Fava, ${ }^{58 b, 58 c}$ F. Feldbauer, ${ }^{4}$ G. Felici, ${ }^{23 a}$ C. Q. Feng, ${ }^{55,43}$ M. Fritsch ${ }^{4}$ C. D. Fu, ${ }^{1}$ Y. Fu, ${ }^{1}$ Q. Gao, ${ }^{1}$ X. L. Gao, ${ }^{55,43}$ Y. Gao, ${ }^{45}$ Y. Gao, ${ }^{56}$ Y. G. Gao, ${ }^{6}$ Z. Gao,${ }^{55,43}$ B. Garillon, ${ }^{26}$ I. Garzia, ${ }^{24 a}$ E. M. Gersabeck, ${ }^{50}$ A. Gilman, ${ }^{51}$ K. Goetzen, ${ }^{11}$ L. Gong, ${ }^{34}$ W. X. Gong, ${ }^{1,43}$ W. Gradl, ${ }^{26}$ M. Greco, ${ }^{58 a, 58 c}$ L. M. Gu, ${ }^{33}$ M. H. Gu, ${ }^{1,43}$ S. Gu, ${ }^{2}$ Y. T. Gu, ${ }^{13}$ A. Q. Guo, ${ }^{22}$ L. B. Guo, ${ }^{32}$ R. P. Guo, ${ }^{36}$ Y. P. Guo ${ }^{26}$ A. Guskov, ${ }^{27}$ S. Han ${ }^{60}$ X. Q. Hao,${ }^{16}$ F. A. Harris, ${ }^{48}$ K. L. He, ${ }^{1,47}$ F. H. Heinsius, ${ }^{4}$ T. Held, ${ }^{4}$ Y. K. Heng, ${ }^{1,43,47}$ M. Himmelreich, ${ }^{1, \mathrm{~g}}$ Y. R. Hou, ${ }^{47}$ Z. L. Hou, ${ }^{1}$ H. M. Hu, ${ }^{1,47}$ J. F. Hu, ${ }^{38, \mathrm{~h}}$ T. Hu, ${ }^{1,43,47}$ Y. Hu, ${ }^{1}$ G. S. Huang, ${ }^{5,43}$ J. S. Huang, ${ }^{16}$ X. T. Huang, ${ }^{37}$ X. Z. Huang, ${ }^{33}$ N. Huesken, ${ }^{52}$ T. Hussain, ${ }^{57}$ W. Ikegami Andersson, ${ }^{59}$ W. Imoehl ${ }^{22}$ M. Irshad, ${ }^{55,43}$ Q. Ji, ${ }^{1}$ Q. P. Ji, ${ }^{16}$ X. B. Ji, ${ }^{1,47}$ X. L. Ji, ${ }^{1,43}$ H. L. Jiang, ${ }^{37}$ X. S. Jiang, ${ }^{1,43,47}$ X. Y. Jiang, ${ }^{34}$ J. B. Jiao, ${ }^{37}$ Z. Jiao, ${ }^{18}$ D. P. Jin, ${ }^{1,43,47}$ S. Jin ${ }^{33}$ Y. Jin ${ }^{49}$ T. Johansson, ${ }^{59}$ N. Kalantar-Nayestanaki, ${ }^{29}$ X. S. Kang, ${ }^{31}$ R. Kappert, ${ }^{29}$ M. Kavatsyuk, ${ }^{29}$ B. C. Ke, ${ }^{1}$ I. K. Keshk, ${ }^{4}$ A. Khoukaz, ${ }^{52}$ P. Kiese, ${ }^{26}$ R. Kiuchi, ${ }^{1}$ R. Kliemt, ${ }^{11}$ L. Koch, ${ }^{28}$ O. B. Kolcu, ${ }^{46 b, f}$ B. Kopf, ${ }^{4}$ M. Kuemmel, ${ }^{4}$ M. Kuessner, ${ }^{4}$ A. Kupsc,${ }^{59}$ M. Kurth, ${ }^{1}$ M. G. Kurth,${ }^{1,47}$ W. Kühn, ${ }^{28}$ J. S. Lange, ${ }^{28}$

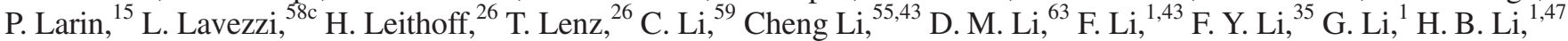
H. J. Li ${ }^{9, j}$ J. C. Li, ${ }^{1}$ J. W. Li ${ }^{41}$ Ke Li, ${ }^{1}$ L. K. Li ${ }^{1}{ }^{2}$ Lei Li,${ }^{3}$ P. L. Li,${ }^{55,43}$ P. R. Li,${ }^{30}$ Q. Y. Li,${ }^{37}$ W. D. Li,${ }^{1,47}$ W. G. Li ${ }^{1}$ X. H. Li ${ }^{55,43}$ X. L. Li ${ }^{37}$ X. N. Li,${ }^{1,43}$ Z. B. Li, ${ }^{44}$ Z. Y. Li ${ }^{44}$ H. Liang, ${ }^{1,47}$ H. Liang, ${ }^{55,43}$ Y. F. Liang, ${ }^{40}$ Y. T. Liang, ${ }^{28}$

G. R. Liao, ${ }^{12}$ L. Z. Liao, ${ }^{1,47}$ J. Libby, ${ }^{21}$ C. X. Lin,${ }^{44}$ D. X. Lin, ${ }^{15}$ Y. J. Lin, ${ }^{13}$ B. Liu, ${ }^{38, h}$ B. J. Liu, ${ }^{1}$ C. X. Liu, ${ }^{1}$ D. Liu, ${ }^{55,43}$

D. Y. Liu, ${ }^{38, h}$ F. H. Liu ${ }^{39}$ Fang Liu, ${ }^{1}$ Feng Liu, ${ }^{6}$ H. B. Liu, ${ }^{13}$ H. M. Liu ${ }^{1,47}$ Huanhuan Liu, ${ }^{1}$ Huihui Liu, ${ }^{17}$ J. B. Liu, ${ }^{55,43}$ J. Y. Liu, ${ }^{1,47}$ K. Y. Liu, ${ }^{31}$ Ke Liu, ${ }^{6}$ L. Y. Liu, ${ }^{13}$ Q. Liu, ${ }^{47}$ S. B. Liu, ${ }^{55,43}$ T. Liu, ${ }^{1,47}$ X. Liu, ${ }^{30}$ X. Y. Liu, ${ }^{1,47}$ Y. B. Liu, ${ }^{34}$ Z. A. Liu, ${ }^{1,43,47}$ Zhiqing Liu, ${ }^{37}$ Y. F. Long, ${ }^{35}$ X. C. Lou, ${ }^{1,43,47}$ H. J. Lu, ${ }^{18}$ J. D. Lu, ${ }^{1,47}$ J. G. Lu, ${ }^{1,43}$ Y. Lu, ${ }^{1}$ Y. P. Lu, ${ }^{1,43}$ C. L. Luo, ${ }^{32}$ M. X. Luo, ${ }^{62}$ P. W. Luo, ${ }^{44}$ T. Luo, ${ }^{9, j}$ X. L. Luo, ${ }^{1,43}$ S. Lusso, ${ }^{58 c}$ X. R. Lyu, ${ }^{47}$ F. C. Ma ${ }^{31}$ H. L. Ma, ${ }^{1}$ L. L. Ma, ${ }^{37}$ M. M. Ma ${ }^{1,47}$ Q. M. Ma, X. N. Ma ${ }^{14}$ X.X. Ma ${ }^{1,47}$ X. Y. Ma, ${ }^{1,43}$ Y. M. Ma ${ }^{37}$ F. E. Maas, ${ }^{15}$ M. Maggiora, ${ }^{58 a, 58 c}$ S. Maldaner, ${ }^{26}$ S. Malde, ${ }^{53}$ Q. A. Malik, ${ }^{57}$ A. Mangoni, ${ }^{23 b}$ Y. J. Mao, ${ }^{35}$ Z. P. Mao, ${ }^{1}$ S. Marcello, ${ }^{58 a, 58 c}$ Z. X. Meng, ${ }^{49}$ J. G. Messchendorp, ${ }^{29}$ G. Mezzadri, ${ }^{24 a}$ J. Min, ${ }^{1,43}$ T. J. Min, ${ }^{33}$ R. E. Mitchell, ${ }^{22}$ X. H. Mo, ${ }^{1,43,47}$ Y. J. Mo, ${ }^{6}$

C. Morales Morales, ${ }^{15}$ N. Yu. Muchnoi,${ }^{10, d}$ H. Muramatsu, ${ }^{51}$ A. Mustafa,${ }^{4}$ S. Nakhoul,${ }^{11, g}$ Y. Nefedov,${ }^{27}$ F. Nerling, ${ }^{11, g}$ I. B. Nikolaev ${ }^{10, d}$ Z. Ning, ${ }^{1,43}$ S. Nisar, ${ }^{8, k}$ S. L. Niu, ${ }^{1,43}$ S. L. Olsen, ${ }^{47}$ Q. Ouyang, ${ }^{1,43,47}$ S. Pacetti, ${ }^{23 b}$ Y. Pan, ${ }^{55,43}$ M. Papenbrock ${ }^{59}$ P. Patteri, ${ }^{23 a}$ M. Pelizaeus, ${ }^{4}$ H. P. Peng, ${ }^{55,43}$ K. Peters, ${ }^{11, g}$ J. Pettersson, ${ }^{59}$ J. L. Ping, ${ }^{32}$ R. G. Ping, ${ }^{1,47}$ A. Pitka, ${ }^{4}$ R. Poling,${ }^{51}$ V. Prasad,${ }^{55,43}$ H. R. Qi, ${ }^{2}$ M. Qi,${ }^{33}$ T. Y. Qi ${ }^{2}$ S. Qian, ${ }^{1,43}$ C. F. Qiao, ${ }^{47}$ N. Qin, ${ }^{60}$ X. P. Qin, ${ }^{13}$ X. S. Qin, ${ }^{4}$ Z. H. Qin, ${ }^{1,43}$ J. F. Qiu, ${ }^{1}$ S. Q. Qu ${ }^{34, \dagger}$ K. H. Rashid ${ }^{57,1}$ K. Ravindran, ${ }^{21}$ C. F. Redmer, ${ }^{26}$ M. Richter, ${ }^{4}$ A. Rivetti ${ }^{58 c}$ V. Rodin, ${ }^{29}$ M. Rolo ${ }^{58 c}$ G. Rong, ${ }^{1,47}$ Ch. Rosner, ${ }^{15}$ M. Rump ${ }^{52}$ A. Sarantsev, ${ }^{27, e}$ M. Savrié, ${ }^{24 b}$ Y. Schelhaas, ${ }^{26}$ K. Schoenning, ${ }^{59}$ W. Shan, ${ }^{19}$ X. Y. Shan, ${ }^{55,43}$ M. Shao, ${ }^{55,43}$ C. P. Shen, ${ }^{2}$ P. X. Shen ${ }^{34}$ X. Y. Shen,${ }^{1,47}$ H. Y. Sheng, ${ }^{1}$ X. Shi, ${ }^{1,43}$ X. D. Shi, ${ }^{55,43}$ J. J. Song, ${ }^{37}$ Q. Q. Song, ${ }^{55,43}$ X. Y. Song, ${ }^{1}$ S. Sosio,${ }^{58,58 c}$ C. Sowa ${ }^{4}$ S. Spataro ${ }^{58 a, 58 c}$ F. F. Sui, ${ }^{37}$ G. X. Sun, ${ }^{1}$ J. F. Sun, ${ }^{16}$ L. Sun, ${ }^{60}$ S. S. Sun, ${ }^{1,47}$ X. H. Sun, ${ }^{1}$ Y. J. Sun, ${ }^{55,43}$ Y. K. Sun, ${ }^{55,43}$ Y. Z. Sun, ${ }^{1}$ Z. J. Sun, ${ }^{1,43}$ Z. T. Sun, ${ }^{1}$ Y. T. Tan,${ }^{55,43}$ C. J. Tang, ${ }^{40}$ G. Y. Tang, ${ }^{1}$ X. Tang, ${ }^{1}$ V. Thoren, ${ }^{59}$ B. Tsednee, ${ }^{25}$ I. Uman, ${ }^{46 d}$ B. Wang, ${ }^{1}$ B. L. Wang ${ }^{47}$ C. W. Wang, ${ }^{33}$ D. Y. Wang, ${ }^{35}$ K. Wang, ${ }^{1,43}$ L. L. Wang, ${ }^{1}$ L. S. Wang, ${ }^{1}$ M. Wang, ${ }^{37}$ M. Z. Wang, ${ }^{35}$ Meng Wang, ${ }^{1,47}$

P. L. Wang, ${ }^{1}$ R. M. Wang ${ }^{61}$ W. P. Wang, ${ }^{55,43}$ X. Wang ${ }^{35}$ X. F. Wang, ${ }^{1}$ X. L. Wang, ${ }^{9, j}$ Y. Wang, ${ }^{44}$ Y. Wang,${ }^{55,43}$ Y. F. Wang, ${ }^{1,43,47}$ Y. Q. Wang, ${ }^{1}$ Z. Wang, ${ }^{1,43}$ Z. G. Wang, ${ }^{1,43}$ Z. Y. Wang, ${ }^{1}$ Zongyuan Wang, ${ }^{1,47}$ T. Weber, ${ }^{4}$ D. H. Wei, ${ }^{12}$ J. H. Wei, ${ }^{34}$ P. Weidenkaff, ${ }^{26}$ H. W. Wen, ${ }^{32}$ S. P. Wen, ${ }^{1}$ U. Wiedner, ${ }^{4}$ G. Wilkinson, ${ }^{53}$ M. Wolke, ${ }^{59}$ L. H. Wu, ${ }^{1}$ L. J. Wu,${ }^{1,47}$

Z. Wu, ${ }^{1,43}$ L. Xia,${ }^{55,43}$ Y. Xia, ${ }^{20}$ S. Y. Xiao, ${ }^{1}$ Y. J. Xiao, ${ }^{1,47}$ Z. J. Xiao, ${ }^{32}$ Y. G. Xie, ${ }^{1,43}$ Y. H. Xie, ${ }^{6}$ T. Y. Xing, ${ }^{1,47}$ X. A. Xiong, ${ }^{1,47}$ Q. L. Xiu, ${ }^{1,43}$ G. F. Xu, ${ }^{1}$ J. J. Xu ${ }^{33}$ L. Xu, ${ }^{1}$ Q. J. Xu, ${ }^{14}$ W. Xu, ${ }^{1,47}$ X. P. Xu,${ }^{41}$ F. Yan, ${ }^{56}$ L. Yan, ${ }^{58,58 c}$ W. B. Yan, ${ }^{55,43}$ W. C. Yan, ${ }^{2}$ Y. H. Yan, ${ }^{20}$ H. J. Yang ${ }^{38, h}$ H. X. Yang, ${ }^{1}$ L. Yang, ${ }^{60}$ R. X. Yang, ${ }^{55,43}$ S. L. Yang, ${ }^{1,47}$ Y. H. Yang,${ }^{33}$ Y.X. Yang, ${ }^{12}$ Yifan Yang, ${ }^{1,47}$ Z. Q. Yang, ${ }^{20}$ M. Ye, ${ }^{1,43}$ M. H. Ye, ${ }^{7}$ J. H. Yin, ${ }^{1}$ Z. Y. You, ${ }^{44}$ B. X. Yu,,${ }^{14,47}$ C. X. Yu, ${ }^{34}$ J. S. Yu, ${ }^{20}$ T. Yu, ${ }^{56}$ C. Z. Yuan, ${ }^{1,47}$ X. Q. Yuan, ${ }^{35}$ Y. Yuan, ${ }^{1}$ A. Yuncu, ${ }^{46 b, a}$ A. A. Zafar ${ }^{57}$ Y. Zeng, ${ }^{20}$ B. X. Zhang, ${ }^{1}$ 
B. Y. Zhang, ${ }^{1,43}$ C. C. Zhang, ${ }^{1}$ D. H. Zhang, ${ }^{1}$ H. H. Zhang, ${ }_{14}^{44}$ H. Y. Zhang, ${ }_{45}^{1,43}$ J. Zhang, ${ }_{33}^{1,47}$ J. L. Zhang, ${ }^{61}$ J. Q. Zhang, ${ }^{4}$ J. W. Zhang, ${ }^{1,43,47}$ J. Y. Zhang, ${ }^{1}$ J. Z. Zhang, ${ }^{1,47}$ K. Zhang, ${ }^{1,47}$ L. Zhang, ${ }^{45}$ L. Zhang, ${ }^{33}$ S. F. Zhang, ${ }^{33}$ T. J. Zhang, ${ }^{38,4}$ X. Y. Zhang, ${ }^{37}$ Y. Zhang, ${ }^{55,43}$ Y. H. Zhang, ${ }^{1,43}$ Y. T. Zhang, ${ }^{55,43}$ Yang Zhang, ${ }^{1}$ Yao Zhang, ${ }_{1}^{1}$ Yi Zhang, ${ }^{9, j}$ Yu Zhang, ${ }^{47}$ Z. H. Zhang, ${ }^{6}$ Z. P. Zhang, ${ }^{55}$ Z. Y. Zhang, ${ }^{60}$ G. Zhao, ${ }^{1}$ J. W. Zhao, ${ }^{1,43}$ J. Y. Zhao, ${ }^{1,47}$ J. Z. Zhao, ${ }^{1,43}$ Lei Zhao ${ }^{55,43}$ Ling Zhao, ${ }^{1}$ M. G. Zhao, ${ }^{34, *}$ Q. Zhao, ${ }^{1}$ S. J. Zhao, ${ }^{63}$ T. C. Zhao, ${ }^{1}$ Y. B. Zhao, ${ }^{1,43}$ Z. G. Zhao, ${ }^{55,43}$ A. Zhemchugov, ${ }^{27, b}$ B. Zheng, ${ }^{56}$ J. P. Zheng, ${ }^{1,43}$ Y. Zheng, ${ }^{35}$ Y. H. Zheng, ${ }^{47}$ B. Zhong, ${ }^{32}$ L. Zhou, ${ }^{1,43}$ L. P. Zhou, ${ }^{1,47}$ Q. Zhou, ${ }^{1,47}$ X. Zhou ${ }^{60}$ X. K. Zhou, ${ }^{47}$ X. R. Zhou, ${ }^{55,43}$ Xiaoyu Zhou, ${ }^{20}$ Xu Zhou, ${ }^{20}$ A. N. Zhu, ${ }^{1,47}$ J. Zhu, ${ }^{34}$ J. Zhu, ${ }^{44}$ K. Zhu, ${ }^{1}$ K. J. Zhu, ${ }^{1,43,47}$ S. H. Zhu, ${ }^{54}$ W. J. Zhu, ${ }^{34}$ X. L. Zhu, ${ }^{45}$ Y. C. Zhu, ${ }^{55,43}$ Y. S. Zhu, ${ }^{1,47}$ Z. A. Zhu, ${ }^{1,47}$ J. Zhuang, ${ }^{1,43}$ B. S. Zou, ${ }^{1}$ and J. H. Zou ${ }^{1}$

\title{
(BESIII Collaboration)
}

\author{
${ }^{1}$ Institute of High Energy Physics, Beijing 100049, People's Republic of China \\ ${ }^{2}$ Beihang University, Beijing 100191, People's Republic of China \\ ${ }^{3}$ Beijing Institute of Petrochemical Technology, Beijing 102617, People's Republic of China \\ ${ }^{4}$ Bochum Ruhr-University, D-44780 Bochum, Germany \\ ${ }^{5}$ Carnegie Mellon University, Pittsburgh, Pennsylvania 15213, USA \\ ${ }^{6}$ Central China Normal University, Wuhan 430079, People's Republic of China \\ ${ }^{7}$ China Center of Advanced Science and Technology, Beijing 100190, People's Republic of China \\ ${ }^{8}$ COMSATS University Islamabad, Lahore Campus, Defence Road, Off Raiwind Road, \\ 54000 Lahore, Pakistan \\ ${ }^{9}$ Fudan University, Shanghai 200443, People's Republic of China \\ ${ }^{10}$ G.I. Budker Institute of Nuclear Physics SB RAS (BINP), Novosibirsk 630090, Russia \\ ${ }^{11}$ GSI Helmholtzcentre for Heavy Ion Research GmbH, D-64291 Darmstadt, Germany \\ ${ }^{12}$ Guangxi Normal University, Guilin 541004, People's Republic of China \\ ${ }^{13}$ Guangxi University, Nanning 530004, People's Republic of China \\ ${ }^{14}$ Hangzhou Normal University, Hangzhou 310036, People's Republic of China \\ ${ }^{15}$ Helmholtz Institute Mainz, Johann-Joachim-Becher-Weg 45, D-55099 Mainz, Germany \\ ${ }^{16}$ Henan Normal University, Xinxiang 453007, People's Republic of China \\ ${ }^{17}$ Henan University of Science and Technology, Luoyang 471003, People's Republic of China \\ ${ }^{18}$ Huangshan College, Huangshan 245000, People's Republic of China \\ ${ }^{19}$ Hunan Normal University, Changsha 410081, People's Republic of China \\ ${ }^{20}$ Hunan University, Changsha 410082, People's Republic of China \\ ${ }^{21}$ Indian Institute of Technology Madras, Chennai 600036, India \\ ${ }^{22}$ Indiana University, Bloomington, Indiana 47405, USA \\ ${ }^{23 a}$ INFN Laboratori Nazionali di Frascati, I-00044, Frascati, Italy \\ ${ }^{23 \mathrm{~b}}$ INFN and University of Perugia, I-06100, Perugia, Italy \\ ${ }^{24 a}$ INFN Sezione di Ferrara, I-44122, Ferrara, Italy \\ ${ }^{24 \mathrm{~b}}$ University of Ferrara, I-44122, Ferrara, Italy \\ ${ }^{25}$ Institute of Physics and Technology, Peace Avenue, 54B, Ulaanbaatar 13330, Mongolia \\ ${ }^{26}$ Johannes Gutenberg University of Mainz, Johann-Joachim-Becher-Weg 45, D-55099 Mainz, Germany \\ ${ }^{27}$ Joint Institute for Nuclear Research, 141980 Dubna, Moscow region, Russia \\ ${ }^{28}$ Justus-Liebig-Universitaet Giessen, II. Physikalisches Institut, \\ Heinrich-Buff-Ring 16, D-35392 Giessen, Germany \\ ${ }^{29}$ KVI-CART, University of Groningen, NL-9747 AA Groningen, Netherlands \\ ${ }^{30}$ Lanzhou University, Lanzhou 730000, People's Republic of China \\ ${ }^{31}$ Liaoning University, Shenyang 110036, People's Republic of China \\ ${ }^{32}$ Nanjing Normal University, Nanjing 210023, People's Republic of China \\ ${ }^{33}$ Nanjing University, Nanjing 210093, People's Republic of China \\ ${ }^{34}$ Nankai University, Tianjin 300071, People's Republic of China \\ ${ }^{35}$ Peking University, Beijing 100871, People's Republic of China \\ ${ }^{36}$ Shandong Normal University, Jinan 250014, People's Republic of China \\ ${ }^{37}$ Shandong University, Jinan 250100, People's Republic of China \\ ${ }^{38}$ Shanghai Jiao Tong University, Shanghai 200240, People's Republic of China \\ ${ }^{39}$ Shanxi University, Taiyuan 030006, People's Republic of China \\ ${ }^{40}$ Sichuan University, Chengdu 610064, People's Republic of China \\ ${ }^{41}$ Soochow University, Suzhou 215006, People's Republic of China \\ ${ }^{42}$ Southeast University, Nanjing 211100, People's Republic of China
}




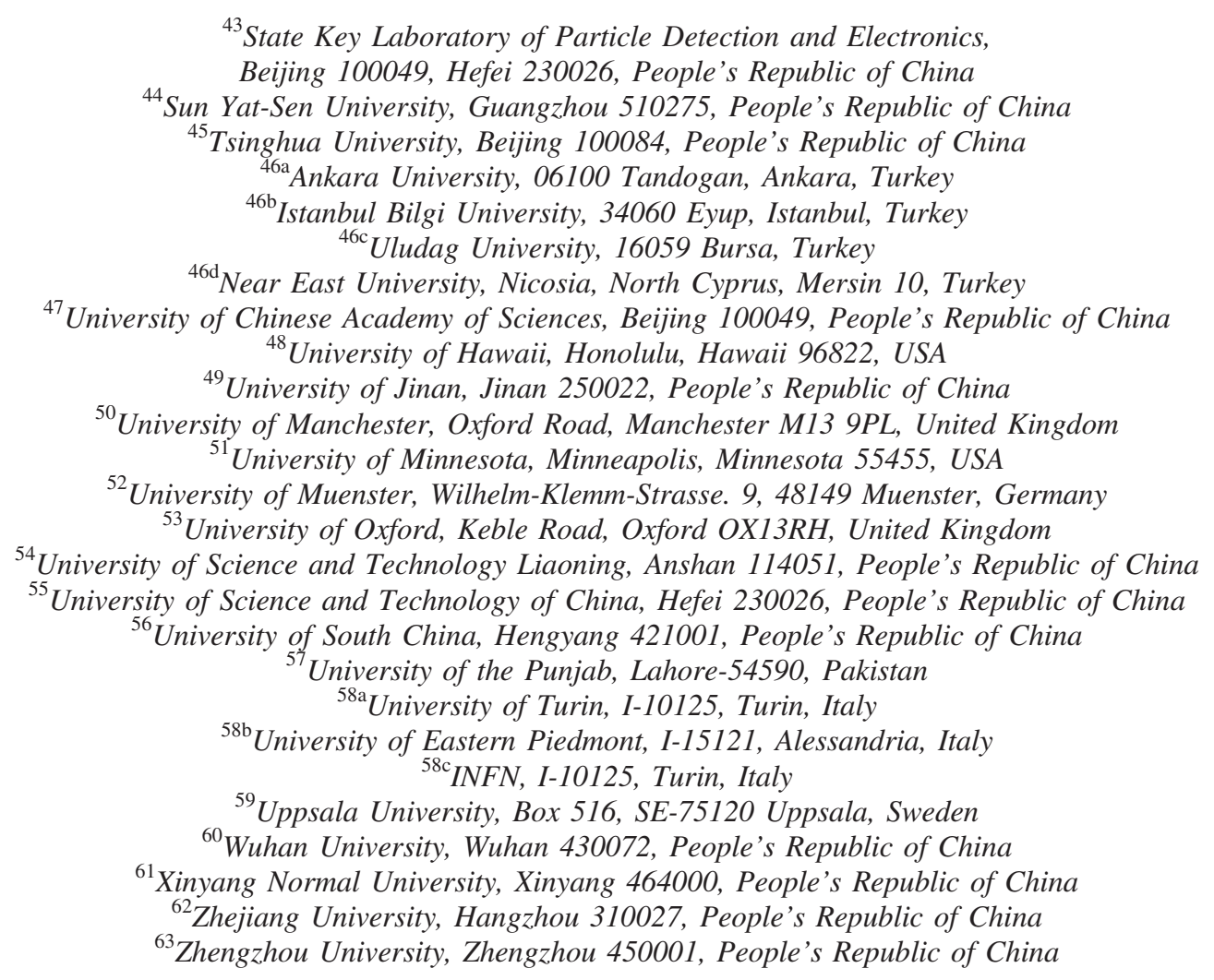

(Received 15 August 2019; published 15 October 2019)

By analyzing $2.93 \mathrm{fb}^{-1}$ of $e^{+} e^{-}$annihilation data taken at the center-of-mass energy $\sqrt{s}=$ $3.773 \mathrm{GeV}$ with the BESIII detector, we determine the branching fractions of the inclusive decays $D^{+} \rightarrow \phi X$ and $D^{0} \rightarrow \phi X$ to be $(1.135 \pm 0.034 \pm 0.031) \%$ and $(1.091 \pm 0.027 \pm 0.035) \%$, respectively, where $X$ denotes any possible particle combination. The first uncertainties are statistical, and the second are systematic. We also determine the branching fractions of the decays $D \rightarrow \phi X$ and their charge conjugate modes $\bar{D} \rightarrow \phi \bar{X}$ separately for the first time, and no significant $C P$ asymmetry is observed.

DOI: 10.1103/PhysRevD.100.072006

\footnotetext{
*Corresponding author. zhaomg@nankai.edu.cn

Corresponding author. qusq@mail.nankai.edu.cn

${ }^{a} A$ lso at Bogazici University, 34342 Istanbul, Turkey.

${ }^{\mathrm{b}}$ Also at the Moscow Institute of Physics and Technology, Moscow 141700, Russia.

${ }^{\mathrm{c}}$ Also at the Functional Electronics Laboratory, Tomsk State University, Tomsk, 634050, Russia.

dAlso at the Novosibirsk State University, Novosibirsk, 630090, Russia.

"Also at the NRC "Kurchatov Institute," PNPI, 188300, Gatchina, Russia.

${ }^{\mathrm{f}}$ Also at Istanbul Arel University, 34295 Istanbul, Turkey.

${ }^{g}$ Also at Goethe University Frankfurt, 60323 Frankfurt am Main, Germany.

${ }^{\mathrm{h}}$ Also at Key Laboratory for Particle Physics, Astrophysics and Cosmology, Ministry of Education; Shanghai Key Laboratory for Particle Physics and Cosmology; Institute of Nuclear and Particle Physics, Shanghai 200240, People's Republic of China.

${ }^{\mathrm{i}}$ Also at Government College Women University, Sialkot 51310. Punjab, Pakistan.

${ }^{\mathrm{j}}$ Also at Key Laboratory of Nuclear Physics and Ion-beam Application (MOE) and Institute of Modern Physics, Fudan University, Shanghai 200443, People's Republic of China.

${ }^{\mathrm{k}}$ Also at Harvard University, Department of Physics, Cambridge, Massachusetts 02138, USA.

Published by the American Physical Society under the terms of the Creative Commons Attribution 4.0 International license. Further distribution of this work must maintain attribution to the author(s) and the published article's title, journal citation, and DOI. Funded by SCOAP.
} 


\section{INTRODUCTION}

Experimental studies of the inclusive $D \rightarrow \phi X$ decays, where $X$ denotes any possible particle combination, are important for charm physics due to the following reasons. First, precise measurements of their branching fractions offer an independent check on the existence of unmeasured or overestimated exclusive decays that include a $\phi$ meson. A measurable difference between the inclusive and exclusive decay branching fractions would indicate the size of as yet unmeasured decays or would imply that some decays are overestimated, requiring complementary or more precise measurements. Previous measurements of the branching fractions for inclusive $D^{+} \rightarrow \phi X$ and $D^{0} \rightarrow \phi X$ decays were made by BES and CLEO [1,2] with 22.3 and $281 \mathrm{pb}^{-1}$ of $e^{+} e^{-}$annihilation data samples taken at the center-of-mass energies $\sqrt{s}=4.03$ and $3.774 \mathrm{GeV}$, respectively. Table I summarizes the branching fractions of the reported exclusive $D$ decays to $\phi$, where the branching fractions of $D^{+} \rightarrow \phi \pi^{+}, D^{0} \rightarrow \phi \pi^{0}$, and $D^{0} \rightarrow \phi \eta$ are quoted from the recent BESIII measurements [3]; the branching fraction of $D^{+} \rightarrow \phi K^{+}$is from the $\mathrm{LHCb}$ measurements [4,5]; while the others are quoted from the particle data group[6]. In this paper, we report improved measurements of the branching fractions of these inclusive decays by using $2.93 \mathrm{fb}^{-1}$ of $e^{+} e^{-}$annihilation data taken at $\sqrt{s}=3.773 \mathrm{GeV}$ with the BESIII detector. Throughout this paper, the charged conjugate modes are implied unless stated explicitly.

Second, charge-parity $(C P)$ violation plays an important role in interpreting the matter-antimatter asymmetry in the Universe and in searching for new physics beyond the standard model (SM). It has been well established in the $K$

TABLE I. The branching fractions of the known exclusive decays $D^{+(0)} \rightarrow \phi X$.

\begin{tabular}{lc}
\hline \hline Decay mode & $\mathcal{B}$ \\
\hline$D^{+} \rightarrow \phi \pi^{+} \pi^{0}$ & $(2.3 \pm 1.0) \%$ \\
$D^{+} \rightarrow \phi \rho^{+}$ & $<1.5 \%$ \\
$D^{+} \rightarrow \phi \pi^{+}$ & $(5.70 \pm 0.14) \times 10^{-3}$ \\
$D^{+} \rightarrow \phi K^{+}$ & $(8.86 \pm 1.14) \times 10^{-6}$ \\
Sum & $(2.87 \pm 1.00) \%$ \\
$D^{0} \rightarrow \phi \gamma$ & $(2.81 \pm 0.19) \times 10^{-5}$ \\
$D^{0} \rightarrow \phi K_{S}^{0}$ & $(4.13 \pm 0.31) \times 10^{-3}$ \\
$D^{0} \rightarrow \phi K_{L}^{0}$ & $(4.13 \pm 0.31) \times 10^{-3}$ \\
$D^{0} \rightarrow \phi \omega$ & $<2.1 \times 10^{-3}$ \\
$D^{0} \rightarrow \phi\left(\pi^{+} \pi^{-}\right)_{\text {S-wave }}$ & $(20 \pm 10) \times 10^{-5}$ \\
$D^{0} \rightarrow\left(\phi \rho^{0}\right)_{\text {S-wave }}$ & $(14.0 \pm 1.2) \times 10^{-4}$ \\
$D^{0} \rightarrow\left(\phi \rho^{0}\right)_{\text {D-wave }}$ & $(8.5 \pm 2.8) \times 10^{-5}$ \\
$D^{0} \rightarrow\left(\phi \rho^{0}\right)_{\text {P-wave }}$ & $(8.1 \pm 3.8) \times 10^{-5}$ \\
$D^{0} \rightarrow \phi \pi^{0}$ & $(1.17 \pm 0.04) \times 10^{-3}$ \\
$D^{0} \rightarrow \phi \eta$ & $(1.81 \pm 0.46) \times 10^{-4}$ \\
Sum & $(1.14 \pm 0.09) \%$ \\
\hline \hline
\end{tabular}

and $B$ meson systems. In the $\mathrm{SM}$, however, $C P$ violation in charm decays is expected to be much smaller [7-9]. Searching for $C P$ violation in $D$ meson decays is important for exploring physics beyond the SM. Recently, $C P$ violation in the charm sector was observed for the first time in the charm hadrons decays at the LHCb [10]. In this paper, we search for $C P$ violation in the inclusive $D \rightarrow \phi X$ and $\bar{D} \rightarrow \phi X$ decays.

\section{BESIII DETECTOR AND MONTE CARLO SIMULATION}

The BESIII detector is a magnetic spectrometer [11] located at the Beijing Electron Positron Collider [12]. The cylindrical core of the BESIII detector consists of a heliumbased multilayer drift chamber, a plastic scintillator timeof-flight system (TOF), and a CsI(Tl) electromagnetic calorimeter (EMC), which are all enclosed in a superconducting solenoidal magnet providing a $1.0 \mathrm{~T}$ magnetic field. The solenoid is supported by an octagonal flux-return yoke with resistive plate counter muon identifier modules interleaved with steel. The acceptance of charged particles and photons is $93 \%$ over a $4 \pi$ solid angle. The chargedparticle momentum resolution at $1 \mathrm{GeV} / c$ is $0.5 \%$, and the $d E / d x$ resolution is $6 \%$ for the electrons from Bhabha scattering. The EMC measures photon energies with a resolution of $2.5 \%(5 \%)$ at $1 \mathrm{GeV}$ in the barrel (end cap) region. The time resolution of the TOF barrel part is $68 \mathrm{ps,}$ while that of the end cap part is 110 ps. The end cap TOF system was upgraded in 2015 with multigap resistive plate chamber technology, providing a time resolution of $60 \mathrm{ps}$ [13]. More details about the design and performance of the detector are given in Ref. [11].

Simulated samples of events produced with the GEANT4based [14] Monte Carlo (MC) package, which includes the geometric description of the BESIII detector and the detector response, are used to determine the detection efficiency and to estimate the backgrounds. The simulation includes the beam energy spread and initial state radiation (ISR) in the $e^{+} e^{-}$annihilations modeled with the generator KKMC $[15,16]$. The inclusive MC samples consist of the production of $D \bar{D}$ pairs with the consideration of quantum coherence for all neutral $D$ modes, the non- $D \bar{D}$ decays of the $\psi(3770)$, the ISR production of the $J / \psi$ and $\psi(3686)$ states, and the continuum processes incorporated in KKMC $[15,16]$. The known decay modes are modeled with EVTGEN $[17,18]$ using branching fractions taken from the Particle Data Group [6], and the remaining unknown charmonium decays are modeled by LUNDCHARM [19]. Final state radiation from charged final state particles is incorporated with the PHOTOS package [20].

\section{ANALYSIS METHOD}

As the $\psi(3770)$ resonance peak lies just above the $D \bar{D}$ threshold, it decays predominately into $D \bar{D}$ meson pairs. 
This advantage is leveraged by using a double-tag method, which was first developed by the MARKIII Collaboration $[21,22]$, to determined absolute branching fractions. If a $\bar{D}$ $\left(D^{-}\right.$or $\left.\bar{D}^{0}\right)$ meson is found in an event, the event is identified as a "single-tag (ST) event." If the partner $D\left(D^{+}\right.$or $\left.D^{0}\right)$ is reconstructed in the rest of the event, the event is identified as a "double-tag (DT) event." In this analysis, the ST $D^{-}$mesons are reconstructed by using $K^{+} \pi^{-} \pi^{-}, K^{+} \pi^{-} \pi^{-} \pi^{0}, K_{S}^{0} \pi^{-}, K_{S}^{0} \pi^{-} \pi^{0}$, and $K_{S}^{0} \pi^{-} \pi^{-} \pi^{+}$, and the ST $\bar{D}^{0}$ mesons are reconstructed by using $K^{+} \pi^{-}$, $K^{+} \pi^{-} \pi^{0}$, and $K^{+} \pi^{-} \pi^{-} \pi^{+}$. The signal $D^{+}$and $D^{0}$ mesons are reconstructed by using $\phi X, \phi \rightarrow K^{+} K^{-}$. The branching fraction for $D \rightarrow \phi X$ decay is given by

$$
\mathcal{B}_{\mathrm{sig}}=\frac{N_{\mathrm{DT}}}{\sum_{i}\left(N_{\mathrm{ST}}^{i} \cdot \epsilon_{\mathrm{DT}}^{i} / \epsilon_{\mathrm{ST}}^{i} / f_{\mathrm{QC}}^{i}\right)}=\frac{N_{\mathrm{DT}}}{\left(N_{\mathrm{ST}} \cdot \epsilon_{\mathrm{sig}}\right)},
$$

where $i$ is the $i$ th ST mode, $N_{\mathrm{DT}}^{i}$ and $N_{\mathrm{ST}}^{i}$ are the yield of the DT and ST events, $\epsilon_{\mathrm{ST}}^{i}$ is the efficiency for reconstructing the tag candidate, and $\epsilon_{\mathrm{DT}}^{i}$ is the efficiency for simultaneously reconstructing the $\bar{D}$ decay to tag mode $i$ and $D$ decay to $\phi X$. $N_{\mathrm{DT}}$ and $N_{\mathrm{ST}}$ are the total yields of the DT and ST events, and $\epsilon_{\mathrm{Sig}}=\sum_{i}\left(N_{\mathrm{ST}}^{i} \cdot \epsilon_{\mathrm{DT}}^{i} / \epsilon_{\mathrm{ST}}^{i} / f_{\mathrm{QC}}^{i}\right) / N_{\mathrm{ST}}$ is the average efficiency of finding the signal decay, weighted by the yields of tag modes in data. Here, $f_{\mathrm{QC}}^{i}$ is a factor to take into account the quantum-correlation (QC) effect in $D^{0} \bar{D}^{0}$ pairs, called QC correction factor. The $f_{\mathrm{QC}}^{i}$ is taken as unity for charged $D$ tags, but determined for neutral $D$ tags following Refs. [23,24] (see the Appendix for more details).

\section{SELECTION AND YIELD OF ST $\bar{D}$ MESONS}

All charged tracks, except those originating from $K_{S}^{0}$ decays, are required to originate in the interaction region, which is defined as $V_{x y}<1 \mathrm{~cm},\left|V_{z}\right|<10 \mathrm{~cm},|\cos \theta|<$ 0.93 , where $V_{x y}$ and $\left|V_{z}\right|$ denote the distances of the closest approach of the reconstructed track to the interaction point perpendicular to and parallel to the beam direction, respectively, and $\theta$ is the polar angle with respect to the beam axis. Charged tracks are identified using confidence levels for the kaon (pion) hypothesis $C L_{K(\pi)}$ [11], calculated with both $d E / d x$ and TOF information. The kaon (pion) candidates are required to satisfy $C L_{K(\pi)}>C L_{\pi(K)}$ and $C L_{K(\pi)}>0$. The $K_{S}^{0}$ candidates are formed from two oppositely charged tracks with $\left|V_{z}\right|<20 \mathrm{~cm}$ and $|\cos \theta|<0.93$. The two charged tracks are assumed to be a $\pi^{+} \pi^{-}$pair without particle identification (PID), and the $\pi^{+} \pi^{-}$invariant mass must be within $(0.487,0.511) \mathrm{GeV} / c^{2}$. The photon candidates are selected from isolated EMC clusters. To suppress electronics noise and beam backgrounds, the clusters are required to have a start time within $700 \mathrm{~ns}$ after the event start time and have an opening angle greater than $10^{\circ}$ with respect to the nearest extrapolated charged track. The energy of each EMC cluster is required to be larger than $25 \mathrm{MeV}$ in the barrel region $(|\cos \theta|<0.8)$ or $50 \mathrm{MeV}$ in the end cap region $(0.86<|\cos \theta|<0.92)$. To select $\pi^{0}$ meson candidates, the $\gamma \gamma$ invariant mass is required to be within $(0.115,0.150) \mathrm{GeV} / c^{2}$. The momentum resolution of $\pi^{0}$ candidates is improved with a kinematic fit that constrains the $\gamma \gamma$ invariant mass to the $\pi^{0}$ nominal mass [6]. For $\bar{D}^{0} \rightarrow K^{+} \pi^{-}$candidates, backgrounds arising from cosmic rays and Bhabha scattering events are rejected with the same requirements as those described in Ref. [25].

Two variables, the energy difference $\Delta E \equiv$ $E_{\bar{D}}-E_{\text {beam }}$ and the beam-energy-constrained mass $M_{\mathrm{BC}} \equiv$ $\sqrt{E_{\text {beam }}^{2} / c^{4}-p_{\bar{D}}^{2} / c^{2}}$, are used to identify the ST $\bar{D}$ candidates. Here, $E_{\text {beam }}$ is the beam energy, and $E_{\bar{D}}\left(p_{\bar{D}}\right)$ is the reconstructed energy (momentum) of the ST $\bar{D}$ candidates in the center-of-mass frame of the $e^{+} e^{-}$system. For a given tag mode, if there are multiple candidates per charm per event, the one with the smallest value of $|\Delta E|$ is retained. Combinatorial backgrounds are suppressed by mode dependent $\Delta E$ requirements, as shown in Table II.

Figure 1 shows the $M_{\mathrm{BC}}$ distributions of the accepted ST $\bar{D}$ candidates. The ST yields $\left(N_{\mathrm{ST}}^{i}\right)$ for different tags are

TABLE II. Summary of the $\Delta E$ requirements, the $M_{\mathrm{BC}}$ signal regions, the ST yields in data $\left(N_{\mathrm{ST}}^{i}\right)$, and the ST efficiencies $\left(\epsilon_{\mathrm{ST}}^{i}\right)$. The uncertainties are statistical only.

\begin{tabular}{lccrr}
\hline \hline Tag mode $i$ & $\Delta E(\mathrm{MeV})$ & $M_{\mathrm{BC}}\left(\mathrm{GeV} / c^{2}\right)$ & $N_{\mathrm{ST}}^{i}$ & $\epsilon_{\mathrm{ST}}^{i}(\%)$ \\
\hline$D^{-} \rightarrow K^{+} \pi^{-} \pi^{-}$ & $(-20,19)$ & $(1.863,1.879)$ & $796040 \pm 1550$ & $50.70 \pm 0.04$ \\
$D^{-} \rightarrow K^{+} \pi^{-} \pi^{-} \pi^{0}$ & $(-53,30)$ & $(1.863,1.879)$ & $239070 \pm 737$ & $24.88 \pm 0.04$ \\
$D^{-} \rightarrow K_{S}^{0} \pi^{-}$ & $(-23,23)$ & $(1.863,1.879)$ & $93258 \pm 312$ & $27.52 \pm 0.12$ \\
$D^{-} \rightarrow K_{S}^{0} \pi^{-} \pi^{0}$ & $(-61,36)$ & $(1.863,1.879)$ & $204591 \pm 553$ & $27.82 \pm 0.08$ \\
$D^{-} \rightarrow K_{S}^{0} \pi^{-} \pi^{-} \pi^{+}$ & $(-20,18)$ & $(1.863,1.879)$ & $111994 \pm 1538$ & $1444953 \pm 2390$ \\
Sum & & & $537047 \pm 762$ & $66.00 \pm 0.06$ \\
$\bar{D}^{0} \rightarrow K^{+} \pi^{-}$ & $(-25,23)$ & $(1.858,1.874)$ & $1075251 \pm 1415$ & $36.25 \pm 0.06$ \\
$\bar{D}^{0} \rightarrow K^{+} \pi^{-} \pi^{0}$ & $(-61,36)$ & $(1.858,1.874)$ & $691228 \pm 952$ & $37.47 \pm 0.05$ \\
$\bar{D}^{0} \rightarrow K^{+} \pi^{-} \pi^{-} \pi^{-}$ & $(-17,15)$ & $(1.858,1.874)$ & $2303526 \pm 1867$ \\
Sum & & & & \\
\hline \hline
\end{tabular}




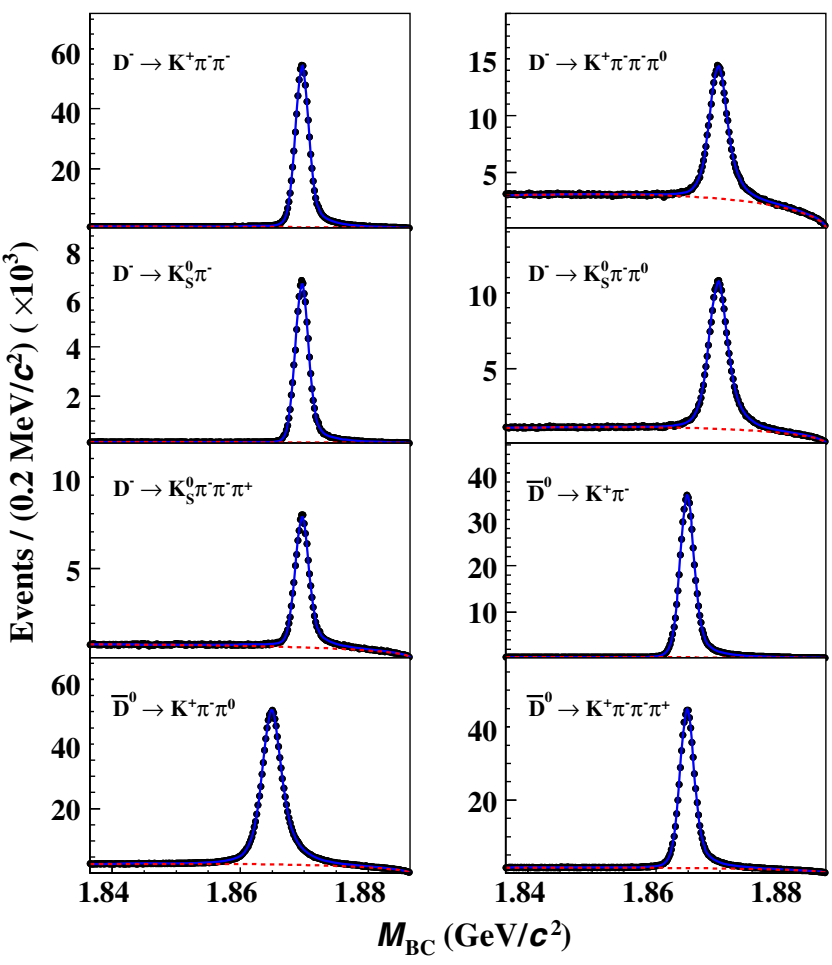

FIG. 1. Fits to the $M_{\mathrm{BC}}$ distributions of the ST $\bar{D}$ meson candidates. The dots with error bars are data, the blue solid curves are the overall fits, and the red dashed curves are the fitted background shapes.

determined using a binned maximum likelihood fit to the corresponding $M_{\mathrm{BC}}$ distribution. A MC-simulated signal shape convolved with a double Gaussian function is used to model the $M_{\mathrm{BC}}$ signal, and the combinatorial backgrounds in $M_{\mathrm{BC}}$ distribution are modeled by an ARGUS function [26] with the end point fixed at $E_{\text {beam. }}$. The ST efficiencies $\left(\epsilon_{\mathrm{ST}}^{i}\right)$ are determined with inclusive MC samples. The ST yields in data within the $\Delta E, M_{\mathrm{BC}}$ signal regions, and the corresponding ST efficiencies are summarized in Table II.

\section{SELECTION AND YIELD OF $D \rightarrow \phi X$}

DT events containing a $\phi$ meson are selected by investigating the system recoiling against the $\mathrm{ST} D^{-}\left(\bar{D}^{0}\right)$. Candidate DT events are required to have at least two

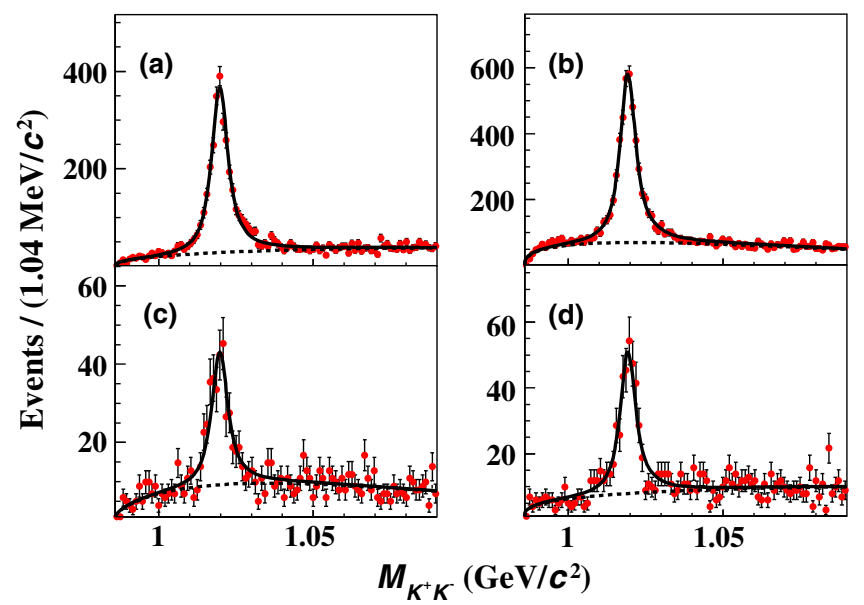

FIG. 2. Fits to the $M_{K^{+} K^{-}}$spectra of the candidate events for (a) $D^{+} \rightarrow \phi X$ and (b) $D^{0} \rightarrow \phi X$ in the $M_{\mathrm{BC}}$ signal region and (c) $D^{+} \rightarrow \phi X$ and (d) $D^{0} \rightarrow \phi X$ in the $M_{\mathrm{BC}}$ sideband region. The dots with error bars are data, the solid curves are the fit results, and the dashed curves are the fitted combinatorial backgrounds.

good charged tracks with opposite charges. The $\phi$ candidates are reconstructed through $\phi \rightarrow K^{+} K^{-}$decays. The selection and identification criteria of the charged kaons are identical to those for the tag side.

The $K^{+} K^{-}$invariant mass $\left(M_{K^{+} K^{-}}\right)$spectra of the accepted candidates for $D \rightarrow \phi X$ in the $M_{\mathrm{BC}}$ signal region are shown in the top row of Fig. 2. The events in the $M_{\mathrm{BC}}$ sideband region, $(1.844,1.860) \mathrm{GeV} / c^{2}$ for $D^{+}$and $(1.840,1.856) \mathrm{GeV} / c^{2}$ for $D^{0}$, are used to estimate the peaking backgrounds in the $M_{K^{+} K^{-}}$spectra, as shown in the bottom row of Fig. 2. For each case, the yield of DT events containing $D \rightarrow \phi X$ signals is obtained by fitting these spectra. A MC-simulated signal shape convolved with a Gaussian function is used to model the $\phi$ signal, and the combinatorial backgrounds are modeled by a reversed ARGUS background function [26]. The sideband contributions are normalized to the same background areas in the $M_{\mathrm{BC}}$ signal region. The fit results are also shown in Fig. 2. The fitted DT yields in the $M_{\mathrm{BC}}$ signal and sideband regions in the data, $N_{\mathrm{DT}}^{\mathrm{sig}}$ and $N_{\mathrm{DT}}^{\mathrm{sid}}$, are given in Table III. The background-subtracted DT yields are calculated by $N_{\mathrm{DT}}^{\mathrm{net}}=N_{\mathrm{DT}}^{\mathrm{sig}}-f_{\mathrm{co}} N_{\mathrm{DT}}^{\mathrm{sid}}$, where $f_{\mathrm{co}}$ is the ratio of the

TABLE III. Summary of the fitted DT yields in the $M_{\mathrm{BC}}$ signal and sideband regions ( $N_{\mathrm{DT}}^{\mathrm{sig}}$ and $\left.N_{\mathrm{DT}}^{\mathrm{sid}}\right)$, background-subtracted DT yields $\left(N_{\mathrm{DT}}^{\text {net }}\right)$, signal efficiencies $\left(\epsilon_{\mathrm{sig}}\right)$, and the measured branching fractions $(\mathcal{B})$. The uncertainties are statistical only.

\begin{tabular}{|c|c|c|c|c|c|c|}
\hline Decay mode & $N_{\mathrm{ST}}^{\text {tot }}$ & $N_{\mathrm{DT}}^{\mathrm{sig}}$ & $N_{\mathrm{DT}}^{\mathrm{sid}}$ & $N_{\text {DT }}^{\text {net }}$ & $\epsilon_{\text {sig }}(\%)$ & $\mathcal{B}(\%)$ \\
\hline$D^{+} \rightarrow \phi X$ & $721005 \pm 1673$ & $1478 \pm 50$ & $153 \pm 18$ & $1352 \pm 53$ & $16.69 \pm 0.20$ & $1.124 \pm 0.045$ \\
\hline$D^{-} \rightarrow \phi X$ & $729840 \pm 1649$ & $1511 \pm 52$ & $155 \pm 18$ & $1384 \pm 55$ & $16.66 \pm 0.20$ & $1.141 \pm 0.046$ \\
\hline$D^{0} \rightarrow \phi X$ & $1152037 \pm 1738$ & $2203 \pm 68$ & $185 \pm 19$ & $2033 \pm 70$ & $16.22 \pm 0.17$ & $1.088 \pm 0.037$ \\
\hline $\bar{D}^{0} \rightarrow \phi X$ & $1146368 \pm 1529$ & $2239 \pm 66$ & $185 \pm 19$ & $2069 \pm 69$ & $16.46 \pm 0.17$ & $1.096 \pm 0.037$ \\
\hline$D^{+} / D^{-} \rightarrow \phi X$ & $1444953 \pm 2390$ & $2989 \pm 77$ & $302 \pm 25$ & $2741 \pm 81$ & $16.71 \pm 0.16$ & $1.135 \pm 0.034$ \\
\hline$D^{0} / \bar{D}^{0} \rightarrow \phi X$ & $2303526 \pm 1867$ & $4441 \pm 98$ & $379 \pm 27$ & $4092 \pm 102$ & $16.28 \pm 0.13$ & $1.091 \pm 0.027$ \\
\hline
\end{tabular}


background area in the $M_{\mathrm{BC}}$ signal region over that in the $M_{\mathrm{BC}}$ sideband region and is determined to be 0.82 for the $D^{+}$decay and 0.92 for the $D^{0}$ decay. These results have been verified by analyzing the inclusive MC sample.

\section{BRANCHING FRACTION}

The detection efficiencies are estimated by analyzing exclusive signal MC samples with the same procedure as for analyzing data. For the ST side, all possible subresonances have been included in the MC simulations. For the signal side, all known $D$ meson decays involving $\phi$ have been included in the MC simulations. Especially, to obtain better data/MC agreement, we have readjusted the branching fraction of $D^{+} \rightarrow \phi \pi^{+} \pi^{0}$, which is dominated by $D^{+} \rightarrow \phi \rho^{+}$, to be $0.6 \%$ in the MC simulations. The efficiencies have been corrected by the small differences in $K^{ \pm}$tracking and PID between the data and $\mathrm{MC}$ simulation. To verify the reliability of the detection efficiencies, we compare the $\cos \theta$ and momentum distributions for $\phi, K^{+}$, and $K^{-}$for the selected candidate events in data and MC simulations, as shown in Figs. 3 and 4. Good data-MC agreement is observed. The detection efficiencies and the measured branching fractions for $D \rightarrow \phi X$ are given in Table III.

Most of the systematic uncertainties originating from the ST selection criteria cancel when using the DT method. The systematic uncertainties in these measurements are assigned relative to the measured branching fractions and are discussed below.

The uncertainties due to the $M_{\mathrm{BC}}$ fits are estimated by using alternative signal shapes, varying the bin sizes,

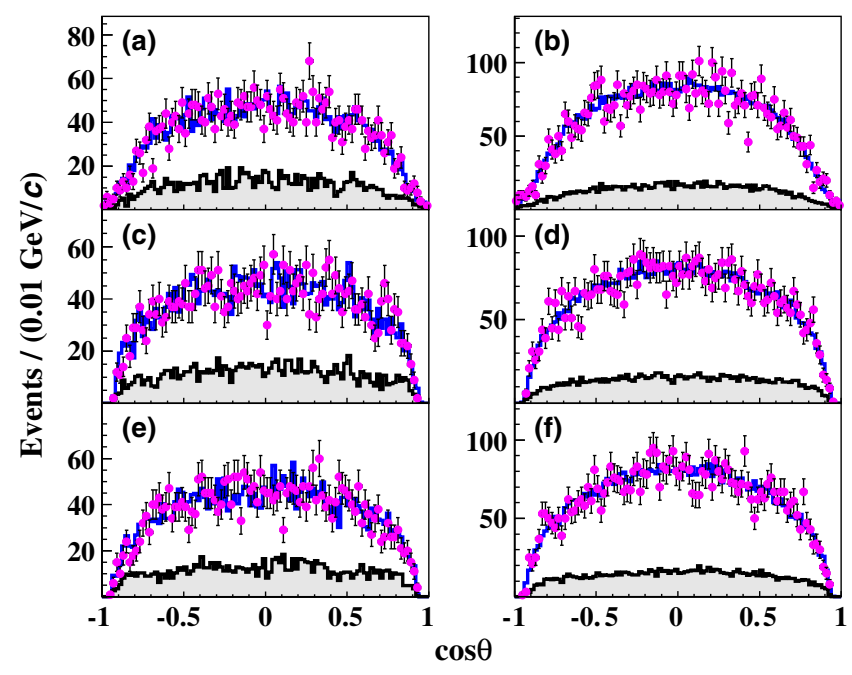

FIG. 3. Comparisons of the $\cos \theta$ distributions for $\phi[(\mathrm{a})$ and (b)], $K^{+}\left[(\mathrm{c})\right.$ and (d)], and $K^{-}$[(e) and (f)] for the candidate events in $D^{+} \rightarrow \phi X$ and $D^{0} \rightarrow \phi X$. The dots with error bars are the data, the solid histograms are the inclusive MC sample, and the gray hatched histograms are the MC-simulated backgrounds. An additional requirement of $\left|M_{K^{+} K^{-}}-1.019\right|<0.02 \mathrm{GeV} / c^{2}$ has been imposed.

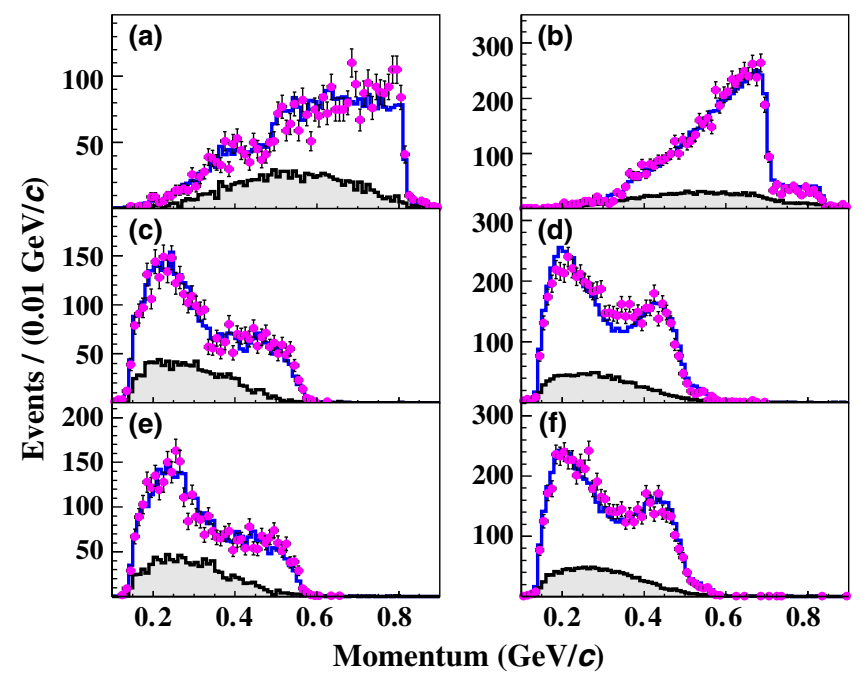

FIG. 4. Comparisons of the momentum distributions for $\phi$ [(a) and (b)], $K^{+}\left[(\mathrm{c})\right.$ and (d)], and $K^{-}[(\mathrm{e})$ and (f)] for the candidate events in $D^{+} \rightarrow \phi X$ and $D^{0} \rightarrow \phi X$. The dots with error bars are the data, the solid histograms are the inclusive MC sample, and the gray hatched histograms are the MC-simulated backgrounds. An additional requirement of $\left|M_{K^{+} K^{-}}-1.019\right|<0.02 \mathrm{GeV} / c^{2}$ has been imposed.

varying the fit ranges, and shifting the end point of the ARGUS background function. We obtain $0.5 \%$ as the total systematic uncertainty due to the $M_{\mathrm{BC}}$ fits.

The tracking and PID efficiencies for $K^{ \pm}$are studied by using DT $D \bar{D}$ hadronic events. In each case, the efficiency to reconstruct a kaon is determined by using the missing mass recoiling against the rest of the event and determining the fraction of events for which the missing kaon can be reconstructed. The differences in the momentum weighted efficiencies between the data and MC simulations (called the data-MC difference) due to tracking and PID are determined to be $(4.2 \pm 0.5) \%$ and $(0.5 \pm 0.5) \%$ per $K^{ \pm}$. After correcting the detection efficiencies obtained by $\mathrm{MC}$ simulations by these differences, the uncertainties of the data-MC differences are assigned as the systematic uncertainties for the $K^{ \pm}$tracking and PID efficiencies. This gives a systematic uncertainty for the $K^{ \pm}$tracking or PID efficiency of $0.5 \%$ per track.

The systematic uncertainties arising from the fit range in the $M_{K^{+} K^{-}}$fits are estimated by a series of fits with alternative intervals. The maximum deviations in the resulting branching fractions are assigned as the associated systematic uncertainties, which are $0.4 \%$ and $1.3 \%$ for $D^{+} \rightarrow \phi X$ and $D^{0} \rightarrow \phi X$, respectively. To estimate the systematic uncertainties due to the signal shape in the $M_{K^{+} K^{-}}$ fits, we use a Breit-Wigner function to describe the $\phi$ signal. The maximum deviations in the resulting branching fractions are assigned as the associated systematic uncertainties, which are $1.6 \%$ and $1.8 \%$ for $D^{+} \rightarrow \phi X$ and $D^{0} \rightarrow \phi X$, respectively. To estimate the systematic uncertainties due to the background shape in the $M_{K^{+} K^{-}}$fits, we use an 
TABLE IV. Systematic uncertainties (in percent) in the measurements of the branching fractions.

\begin{tabular}{lcc}
\hline \hline Source & $D^{+} \rightarrow \phi X$ & $D^{0} \rightarrow \phi X$ \\
\hline$M_{\mathrm{BC}}$ fit & 0.5 & 0.5 \\
$K^{ \pm}$tracking & 1.2 & 1.2 \\
$K^{ \pm}$PID & 1.0 & 1.0 \\
$M_{K^{+} K^{-}}$fit & 1.7 & 2.4 \\
QC effect & $\ldots$ & 0.5 \\
MC statistics & 0.8 & 0.7 \\
Quoted branching fraction & 1.0 & 1.0 \\
Total & 2.7 & 3.2 \\
\hline \hline
\end{tabular}

alternative background shape, $c_{1} \cdot\left(M_{K^{+} K^{-}}-M_{\text {threshold }}\right)^{1 / 2}+$ $c_{3} \cdot\left(M_{K^{+} K^{-}}-M_{\text {threshold }}\right)^{3 / 2}+c_{5} \cdot\left(M_{K^{+} K^{-}}-M_{\text {threshold }}\right)^{5 / 2}$, to describe the background. The maximum deviations in the resulting branching fractions are assigned as the associated systematic uncertainties, which are $0.2 \%$ and $1.6 \%$ for $D^{+} \rightarrow$ $\phi X$ and $D^{0} \rightarrow \phi X$, respectively. We assume that systematic uncertainties arising from the fit range, signal, and background shape are independent and add them in quadrature to obtain the systematic uncertainty of the $M_{K^{+} K^{-}}$fit.

In our nominal analysis, the measured branching fraction of $D^{0} \rightarrow \phi X$ has been corrected by an averaged QC factor $f_{\mathrm{QC}}$ defined in Sec. VI. After this correction, we take the residual uncertainty of $f_{\mathrm{QC}}, 0.5 \%$, as the systematic uncertainty due to the $\mathrm{QC}$ effect. The uncertainties due to limited MC samples are $0.8 \%$ and $0.7 \%$ for $D^{+}$and $D^{0}$ decays, respectively. The uncertainty in the quoted branching fraction of $\phi \rightarrow K^{+} K^{-}$is $1.0 \%$ [6].

Assuming all the sources are independent, the quadratic sum of these uncertainties gives the total systematic uncertainty in the measurement of the branching fraction for each decay. Table IV summarizes the systematic uncertainties in the branching fraction measurements.

\section{ASYMMETRY OF $\mathcal{B}(D \rightarrow \phi X)$ AND $\mathcal{B}(\bar{D} \rightarrow \phi X)$}

We determine the branching fractions of $D \rightarrow \phi X$ and $\bar{D} \rightarrow \phi X$ separately. In this section, charge conjugated modes are not implied. Table III summarizes the ST yields, the DT yields in the $M_{\mathrm{BC}}$ signal and sideband regions, detection efficiencies, and the measured branching fractions. The asymmetry of the branching fractions of $D \rightarrow$ $\phi X$ and $\bar{D} \rightarrow \phi X$ is determined by

$$
\mathcal{A}_{\mathrm{CP}}=\frac{\mathcal{B}(D \rightarrow \phi X)-\mathcal{B}(\bar{D} \rightarrow \phi X)}{\mathcal{B}(D \rightarrow \phi X)+\mathcal{B}(\bar{D} \rightarrow \phi X)} .
$$

The asymmetries for charged and neutral $D \rightarrow \phi X$ decays are determined to be $(-0.7 \pm 2.8 \pm 0.7) \%$ and $(-0.4 \pm$ $2.5 \pm 0.7) \%$, where the uncertainties due to the $M_{\mathrm{BC}}$ fit, $K^{ \pm}$tracking, $K^{ \pm}$PID, $M_{K^{+} K^{-}}$fit, QC effect, and quoted branching fractions in the measurements of $\mathcal{B}(D \rightarrow \phi X)$ and $\mathcal{B}(\bar{D} \rightarrow \phi X)$ cancel. No $C P$ violation is found at the current statistical and systematic precision.

\section{CONCLUSIONS}

By analyzing $2.93 \mathrm{fb}^{-1}$ of $e^{+} e^{-}$annihilation data taken with the BESIII detector at $\sqrt{s}=3.773 \mathrm{GeV}$, the branching fractions of $D^{+} \rightarrow \phi X$ and $D^{0} \rightarrow \phi X$ decays are measured to be $(1.135 \pm 0.034 \pm 0.031) \%$ and $(1.091 \pm 0.027 \pm 0.035) \%$, respectively, where the first uncertainties are statistical and the second are systematic. Comparisons of our results with the previous measurements by CLEO [2] and BES [1] are shown in Table V. Our results are consistent with previous measurements, but with much better precision. These results indicate that the nominal values of the branching fractions for some known exclusive decays of the $D^{+}$meson, e.g., $D^{+} \rightarrow \phi \pi^{+} \pi^{0}$, may be overestimated. Precision measurements of some exclusive $\phi X$ decays of $D^{+}$and $D^{0}$ mesons are required to further understand the discrepancy. We also determine $C P$ asymmetries in the branching fractions of $D \rightarrow \phi X$ and $\bar{D} \rightarrow \phi X$ decays for the first time, but no $C P$ violation is found.

\section{ACKNOWLEDGMENTS}

The authors are thankful for helpful discussions with Professor Xueqian Li, Professor Maozhi Yang, and Doctor Haokai Sun. The BESIII Collaboration thanks the staff of Beijing Electron Positron Collider and the IHEP computing center for their strong support. This work is supported in part by National Key Basic Research Program of China under Contract No. 2015CB856700; National Natural Science Foundation of China (NSFC) under Contracts No. 11875170, No. 11775230, No. 11475090, No. 11335008, No. 11425524, No. 11625523, No. 11635010, and No. 11735014; National Natural Science Foundation of China (NSFC) under Contract No. 11835012; the Chinese Academy of Sciences (CAS) Large-Scale Scientific Facility Program; Joint Large-Scale Scientific Facility Funds of the NSFC and CAS under Contracts No. U1832207, No. U1532257, No. U1532258, and No. U1732263; CAS Key Research Program of Frontier Sciences under Contracts No. QYZDJ-SSWSLH003 and No. QYZDJ-SSW-SLH040; 100 Talents

TABLE V. Comparisons of our branching fractions with the CLEO and BES results (in percent).

\begin{tabular}{lccc}
\hline \hline & This work & CLEO [2] & BES [1] \\
\hline$D^{+} \rightarrow \phi X$ & $1.135 \pm 0.034 \pm 0.031$ & $1.03 \pm 0.10 \pm 0.07$ & $<1.8(90 \%$ C.L.) \\
$D^{0} \rightarrow \phi X$ & $1.091 \pm 0.027 \pm 0.035$ & $1.05 \pm 0.08 \pm 0.07$ & $1.71_{-0.71}^{+0.76} \pm 0.17$ \\
\hline \hline
\end{tabular}


Program of CAS; Institute for Nuclear Physics, Astronomy and Cosmology (INPAC) and Shanghai Key Laboratory for Particle Physics and Cosmology; German Research Foundation DFG under Collaborative Research Center under Contracts No. CRC 1044 and No. FOR 2359; Istituto Nazionale di Fisica Nucleare, Italy; Koninklijke Nederlandse Akademie van Wetenschappen (KNAW) under Contract No. 530-4CDP03; Ministry of Development of Turkey under Contract No. DPT2006K120470; National Science and Technology fund; the Knut and Alice Wallenberg Foundation (Sweden) under Contract No. 2016.0157; the Royal Society, U.K., under Contract No. DH160214; the Swedish Research Council; U.S. Department of Energy under Contracts No. DE-FG0205ER41374, No. DE-SC-0010118, and No. DE-SC0012069; University of Groningen (RuG) and the Helmholtzzentrum fuer Schwerionenforschung $\mathrm{GmbH}$ (GSI), Darmstadt.

\section{APPENDIX: QC CORRECTION FACTOR}

At $\psi(3770)$, the $D^{0} \overline{D^{0}}$ pairs are produced coherently. The impact of the QC effect on the measurement of the branching fraction of $D^{0} \rightarrow \phi X$ is considered by two aspects: the strong-phase parameters of the tag modes and the $C P+$ fraction of the $D^{0} \rightarrow \phi X$ decay.

\section{Formulas}

Due to the QC effect, the yield of the $i$ th ST candidates can be written as $[23,24]$

$$
N_{\mathrm{ST}}^{i}=\left(1+R_{\mathrm{WS}, f}^{i}\right) \cdot 2 N_{D^{0} \bar{D}^{0}} \cdot \mathcal{B}_{\mathrm{ST}}^{i} \cdot \epsilon_{\mathrm{ST}}^{i},
$$

and the yield of the DT candidates, i.e., $C P \pm$ eigenstate decay vs the $i$ th tag, can be written as

$$
\begin{aligned}
N_{\mathrm{DT}}^{i}= & \left(1+R_{\mathrm{WS}, f}^{i} \mp r_{f}^{i} z_{f}^{i}\right) \cdot 2 N_{D^{0} \bar{D}^{0}} \\
& \cdot \mathcal{B}_{\mathrm{ST}}^{i} \cdot \mathcal{B}_{\mathrm{sig}}^{i} \cdot \epsilon_{\mathrm{DT}}^{i},
\end{aligned}
$$

where $N_{D^{0} \bar{D}^{0}}$ is the total number of $D^{0} \overline{D^{0}}$ pairs produced in data; $\epsilon_{\mathrm{ST}(\mathrm{DT})}^{i}$ is the efficiency of reconstructing the ST (DT) candidates; $\mathcal{B}_{\mathrm{ST}}^{i}$ and $\mathcal{B}_{\text {sig }}^{i}$ are the branching fractions of the $\mathrm{ST}$ and signal decays, respectively; $R_{\mathrm{WS}, f}^{i}$ is the ratio of the Cabibbo-suppressed and Cabibbo-favored rates; $r_{f}^{i}$ is defined as $r_{f}^{i} e^{-i \delta_{f}^{i}} \equiv \frac{\left\langle f \mid \overline{D^{0}}\right\rangle}{\left\langle f \mid D^{0}\right\rangle} ; z_{f}^{i}$ is defined as $z_{f}^{i} \equiv 2 \cos \delta_{f}^{i}$; and $\delta_{f}^{i}$ is the strong-phase difference between these two amplitudes.

In this analysis, $R_{\mathrm{WS}, f}^{i}$ is taken to be $r_{i}^{2}$, where $r_{i}$ is the ratio of the Cabibbo-suppressed and Cabibbo-favored amplitudes for $D^{0} \overline{D^{0}}$ decays to same final state. Then, we have

$$
\begin{aligned}
N_{\mathrm{ST}}^{i}= & \left(1+r_{i}^{2}\right) \cdot 2 N_{D^{0} \bar{D}^{0}} \cdot \mathcal{B}_{\mathrm{ST}}^{i} \cdot \epsilon_{\mathrm{ST}}^{i}, \\
N_{\mathrm{DT}}^{i}= & \left(1+r_{i}^{2} \mp 2 r_{i} R_{i} \cos \delta_{f}^{i}\right) \cdot 2 N_{D^{0} \bar{D}^{0}} \\
& \cdot \mathcal{B}_{\mathrm{ST}}^{i} \cdot \mathcal{B}_{\mathrm{sig}}^{i} \cdot \epsilon_{\mathrm{DT}}^{i},
\end{aligned}
$$

where $R_{i}$ is the coherence factor, $0<R_{i} \leq 1$, that quantifies the dilution due to integrating over the phase space (for $D \rightarrow K^{ \pm} \pi^{\mp}, R=1.00$ ) [27,28].

According to Eqs. (A3) and (A4), the absolute branching fraction for the signal decay is calculated by

$$
\mathcal{B}_{\mathrm{Sig}}^{i}=\frac{1}{1 \mp C_{f}^{i}} \cdot \frac{N_{\mathrm{DT}}^{i}}{N_{\mathrm{ST}}^{i} \cdot\left(\epsilon_{\mathrm{DT}}^{i} / \epsilon_{\mathrm{ST}}^{i}\right)},
$$

where $C_{f}^{i}$ is the strong-phase factor, which can be calculated by

$$
C_{f}^{i}=\frac{2 r_{i} R_{i} \cos \delta_{f}^{i}}{1+r_{i}^{2}} .
$$

The amplitude of the neutral $D$ decays can be decomposed as mixture of the $C P+$ and $C P-$ components. This gives $F_{+}^{\text {sig }}=1-F_{-}^{\text {sig }}$, where $F_{+}^{\text {sig }}$ and $F_{-}^{\text {sig }}$ are the $C P+$ and $C P-$ fractions of the decay, respectively. The yield of the DT candidates tagged by the Cabibbo-favored tag mode $i$ can be written as

$$
\begin{aligned}
N_{\mathrm{DT}}^{i}= & F_{+}^{\mathrm{sig}} \cdot\left(1+r_{i}^{2}\right) \cdot\left(1-C_{f}^{i}\right) \cdot 2 N_{D^{0} \bar{D}^{0}} \\
& \cdot \mathcal{B}_{\mathrm{ST}}^{i} \cdot \mathcal{B}_{\mathrm{sig}}^{i} \cdot \epsilon_{\mathrm{DT}}^{i}+F_{-}^{\mathrm{sig}} \cdot\left(1+r_{i}^{2}\right) \cdot\left(1+C_{f}^{i}\right) \\
& \cdot 2 N_{D^{0} \bar{D}^{0}} \cdot \mathcal{B}_{\mathrm{ST}}^{i} \cdot \mathcal{B}_{\mathrm{sig}}^{i} \cdot \epsilon_{\mathrm{DT}}^{i} \cdot \\
= & {\left[1-C_{f}^{i} \cdot\left(2 F_{+}^{\mathrm{sig}}-1\right)\right] \cdot\left(1+r_{i}^{2}\right) \cdot 2 N_{D^{0} \bar{D}^{0}} } \\
& \cdot \mathcal{B}_{\mathrm{ST}}^{i} \cdot \mathcal{B}_{\mathrm{sig}}^{i} \cdot \epsilon_{\mathrm{DT}}^{i} \cdot
\end{aligned}
$$

According to Eqs. (A3) and (A7), the branching fraction of the signal decay can be calculated by

TABLE VI. Summary of the obtained $C_{f}$ and the parameters used to calculate the strong-phase factors.

\begin{tabular}{lcccc}
\hline \hline ST mode & $r(\%)$ & $R$ & $\delta_{f}\left({ }^{\circ}\right)$ & $C_{f}$ \\
\hline$D \rightarrow K^{ \pm} \pi^{\mp}$ & $5.86 \pm 0.02[29]$ & 1.00 & $194.7_{-17}^{+8.4}[29]$ & $-0.113_{-0.009}^{+0.004}$ \\
$D \rightarrow K^{ \pm} \pi^{\mp} \pi^{0}$ & $4.47 \pm 0.12[28]$ & $0.81 \pm 0.06[28]$ & $198.0_{-15}^{+14}[28]$ & $-0.069_{-0.008}^{+0.008}$ \\
$D \rightarrow K^{ \pm} \pi^{\mp} \pi^{\mp} \pi^{ \pm}$ & $5.49 \pm 0.06[28]$ & $0.43_{-0.13}^{+0.17}[28]$ & $128.0_{-17}^{+28}[28]$ & $-0.029_{-0.014}^{+0.021}$ \\
\hline \hline
\end{tabular}




$$
\begin{aligned}
\mathcal{B}_{\mathrm{sig}}^{i} & =\frac{1}{1-C_{f}^{i} \cdot\left(2 F_{+}^{\mathrm{sig}}-1\right)} \cdot \frac{N_{\mathrm{DT}}^{i}}{N_{\mathrm{ST}}^{i} \cdot\left(\epsilon_{\mathrm{DT}}^{i} / \epsilon_{\mathrm{ST}}^{i}\right)} \\
& =f_{\mathrm{QC}}^{i} \cdot \frac{N_{\mathrm{DT}}^{i}}{N_{\mathrm{ST}}^{i} \cdot\left(\epsilon_{\mathrm{DT}}^{i} / \epsilon_{\mathrm{ST}}^{i}\right)}
\end{aligned}
$$

Here, $f_{\mathrm{QC}}^{i}=\frac{1}{1-C_{f}^{i} \cdot\left(2 F_{+}^{\mathrm{sig}}-1\right)}$ is the QC correction factor to be determined.

\section{Strong-phase factor $C_{f}^{i}$}

Based on Eq. (A6) and quoted parameters of $r_{i}, R_{i}$, and $\delta_{f}^{i}$, we obtain the strong-phase factor $C_{f}^{i}$ for the different ST modes. The quoted parameters of $r_{i}, R_{i}$ and $\delta_{f}^{i}$ as well as the obtained $C_{f}^{i}$ are listed in Table VI.

\section{3. $C P+$ fraction of the signal decay}

According to Ref. [30], the $C P+$ fraction for the signal decay is determined by

$$
F_{+}^{\text {sig }}=\frac{N_{+}}{N_{+}+N_{-}},
$$

in which $N_{ \pm}$is the ratio of the DT and ST yields with $C P \mp$ tags and is obtained by

$$
\begin{aligned}
& N_{ \pm}=\frac{M_{\text {measured }}^{ \pm}}{S^{ \pm}}, \\
& S^{ \pm}=\frac{S_{\text {measured }}^{ \pm}}{1-\eta_{ \pm} y_{D}},
\end{aligned}
$$

where $M^{ \pm}$is the DT yields for $D^{0} \rightarrow \phi X$ vs $C P \mp$ tags and $S^{ \pm}$is the corrected ST yields for the $C P \pm$ decay modes. Here, $\eta_{ \pm}= \pm 1$ for $C P \pm$ decay modes, and $y_{D}$ is the $D^{0} \bar{D}^{0}$ mixing parameter from the heavy flavor averaging group (HFAG) average [6].

To extract $F_{+}^{\text {sig }}$ of the $D^{0} \rightarrow \phi X$ decay, we use the $C P+$ tag of $D \rightarrow K^{+} K^{-}$and the $C P-\operatorname{tag}$ of $D \rightarrow K_{S}^{0} \pi^{0}$. Figures 5 and 6 show the fits to the $M_{\mathrm{BC}}$ distributions of the ST candidates and the $M_{K^{+} K^{-}}$distributions of the DT candidates. From the fits, we obtain the measured ST and DT yields ( $S_{\text {measured }}^{ \pm}$and $M_{\text {measured }}^{ \pm}$), as summarized in Table VII. Inserting these numbers in Eqs. (A9) and (A10), we obtain $F_{+}^{\text {sig }}=0.64 \pm 0.05$.

\section{Impact on the measured branching fraction}

Inserting the $C_{f}^{i}$ and $F_{+}^{\text {sig }}$ obtained above in Eqs. (A6) and (A9), we obtain the QC correction factors for the

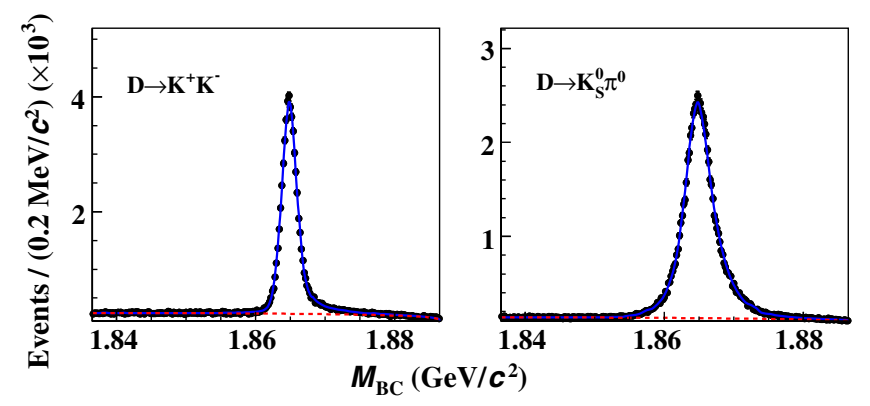

FIG. 5. Fit to the $M_{\mathrm{BC}}$ distributions of the $D \rightarrow K^{+} K^{-}$and $D \rightarrow K_{S}^{0} \pi^{0}$ candidates. The dots with error bars are data, the blue solid curves are the overall fits, and the red dashed curves are the fitted background shapes.

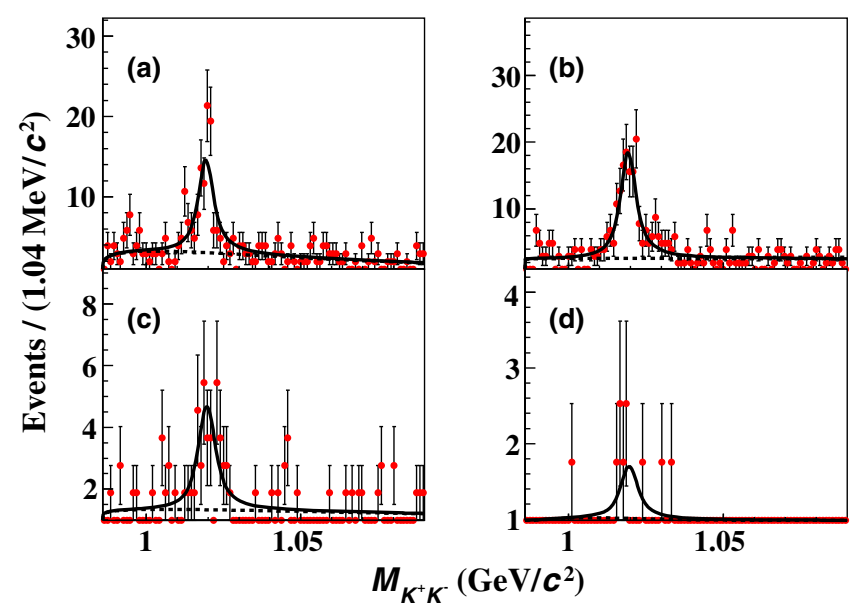

FIG. 6. Fits to the $M_{K^{+} K^{-}}$spectra of the candidate events for $D^{0} \rightarrow \phi X$ tagged by [(a) and (c)] $\overline{D^{0}} \rightarrow K^{+} K^{-}$and [(b) and (d)] $\overline{D^{0}} \rightarrow K_{S}^{0} \pi^{0}$ in the $M_{\mathrm{BC}}$ signal and sideband regions, respectively. The dots with error bars are data, the solid curves are the fit results, and the dashed curves are the fitted combinatorial backgrounds.

$D \rightarrow K^{ \pm} \pi^{\mp}, \quad D \rightarrow K^{ \pm} \pi^{\mp} \pi^{0}$, and $D \rightarrow K^{ \pm} \pi^{\mp} \pi^{\mp} \pi^{ \pm} \quad$ ST decays to be $(96.9 \pm 0.3 \pm 1.1) \%$, $(98.1 \pm 0.3 \pm 0.7) \%$, and $(99.2 \pm 0.7 \pm 0.3) \%$, where the first and second uncertainties are from $C_{f}^{i}$ and $F_{+}^{\mathrm{sig}}$, respectively.

TABLE VII. Summaries of the data yields and the MC efficiencies for the ST and DT candidates.

\begin{tabular}{lcc}
\hline \hline Decay mode & $D \rightarrow K^{+} K^{-}$ & $D \rightarrow K_{S}^{0} \pi^{0}$ \\
\hline$S_{\text {measured }}^{ \pm}$ & $57147 \pm 372$ & $65407 \pm 309$ \\
$M_{\text {measured }}^{ \pm}$ & $73 \pm 15$ & $147 \pm 15$ \\
\hline \hline
\end{tabular}


[1] J. Z. Bai et al. (BES Collaboration), Direct measurement of $\mathrm{B}\left(D^{0} \rightarrow \phi X^{0}\right)$ and $\mathrm{B}\left(D^{+} \rightarrow \phi X^{+} f\right)$, Phys. Rev. D 62, 052001 (2000).

[2] G. S. Huang et al. (CLEO Collaboration), Meaurement of inclusive production of $\eta, \eta^{\prime}$ and $\phi$ mesons in $D^{0}, D^{+}$and $D_{s}^{+}$decays, Phys. Rev. D 74, 112005 (2006).

[3] M. Ablikim et al. (BESIII Collaboration), Measurement of branching fractions for $D$ meson decaying into $\phi$ meson and a pseudoscalar meson, arXiv:1907.11258.

[4] R. Aaij et al. (LHCb Collaboration), Measurement of the branching fractions of the decays $D^{+} \rightarrow K^{-} K^{+} K^{+}, D^{+} \rightarrow$ $\pi^{-} \pi^{+} K^{+}$and $D_{s}^{+} \rightarrow \pi^{-} K^{+} K^{+}$, J. High Energy Phys. 03 (2019) 176.

[5] R. Aaij et al. (LHCb Collaboration), Dalitz plot analysis of the $D^{+} \rightarrow K^{-} K^{+} K^{+}$decay, J. High Energy Phys. 04 (2019) 063.

[6] M. Tanabashi et al. (Particle Data Group), Review of particle physics, Phys. Rev. D 98, 030001 (2018).

[7] M. Golden and B. Grinstein, Enhanced $C P$ violations in hadronic charm decays, Phys. Lett. B 222, 501 (1989).

[8] M. Artuso, B. Meadows, and A. A. Petrov, Charm meson decays, Annu. Rev. Nucl. Part. Sci. 58, 249 (2008).

[9] H. N. Li, C. D. Lu, and F. S. Yu, Branching ratio and direct $C P$ asymmetries in $D \rightarrow P P$ decays, Phys. Rev. D 86, 036012 (2012).

[10] R. Aaij et al. (LHCb Collaboration), Observation of $C P$ Violation in Charm Decays, Phys. Rev. Lett. 122, 211803 (2019).

[11] M. Ablikim et al. (BESIII Collaboration), Design and construction of the BESIII detector, Nucl. Instrum. Methods Phys. Res., Sect. A 614, 345 (2010).

[12] C. H. Yu et al., BEPCII performance and beam dynamics studies on luminosity, Proceedings of IPAC2016, Busan, Korea (JACoW, Geneva, Switzerland, 2016), http:// accelconf.web.cern.ch/AccelConf/ipac2016/doi/JACoWIPAC2016-TUYA01.html.

[13] X. Li et al., Study of MRPC technology for BESIII endcapTOF upgrade, Radiat. Detect. Technol. Methods 1, 13 (2017); Y.X. Guo et al., The study of time calibration for upgraded end cap TOF of BESIII, Radiat. Detect. Technol. Methods 1, 15 (2017).

[14] S. Agostinelli et al. (GEANT4 Collaboration), GEANT4: A simulation toolkit, Nucl. Instrum. Meth. Nucl. Instrum. Methods Phys. Res., Sect. A 506, 250 (2003).

[15] S. Jadach, B. F. L. Ward, and Z. Was, The precision Monte Carlo event generator KK for two-Fermion final states in $e^{+} e^{-}$collisions, Comput. Phys. Commun. 130, 260 (2000).
[16] S. Jadach, B. F. L. Ward, and Z. Was, Coherent exclusive exponentiation for precision Monte Carlo calculations, Phys. Rev. D 63, 113009 (2001).

[17] R. G. Ping, Event generators at BESIII, Chin. Phys. C 32, 599 (2008).

[18] D. J. Lange, The EvtGen particle decay simulation package, Nucl. Instrum. Methods Phys. Res., Sect. A 462, 152 (2001).

[19] J. C. Chen, G. S. Huang, X. R. Qi, D. H. Zhang, and Y. S. Zhu, Event generator for $J / \psi$ and $\psi(2 \mathrm{~S})$ decay, Phys. Rev. D 62, 034003 (2000).

[20] E. Richter-Was, QED Bremsstrahlung in semileptonic B and Leptonic $\tau$ decays, Phys. Lett. B 303, 163 (1993).

[21] R. M. Baltrusaitis et al. (MARK-III Collaboration), Direct Measurements of Charmed d Meson Hadronic Branching Fractions, Phys. Rev. Lett. 56, 2140 (1986).

[22] J. Adler et al. (MARK-III Collaboration), A Reanalysis of Charmed d Meson Branching Fractions, Phys. Rev. Lett. 60, 89 (1988).

[23] Z. Z. Xing, $D^{0}-\overline{D^{0}}$ Mixing and $C P$ violation in neutral $D$ meson decays, Phys. Rev. D 55, 196 (1997).

[24] D. M. Asner and W. M. Sun, Time-independent measurements of $D^{0}-\bar{D}^{0}$ mixing and relative strong phases using quantum correlations, Phys. Rev. D 73, 034024 (2006); Erratum, Phys. Rev. D 77, 019901(E) (2008).

[25] M. Ablikim et al. (BESIII Collaboration), Measurement of the $D \rightarrow K^{-} \pi^{+}$strong phase difference in $\psi(3770) \rightarrow$ $D^{0} \overline{D^{0}}$, Phys. Lett. B 734, 227 (2014).

[26] H. Albrecht et al. (ARGUS Collaboration), Search for hadronic $b \rightarrow u$ decays, Phys. Lett. B 241, 278 (1990).

[27] T.Gershon, J. Libby, and G. Wilkinson, Contributions to the width difference in the neutral $D$ system from hadronic decays, Phys. Lett. B 750, 338 (2015).

[28] T. Evans, S. T. Harnew, J. Libby, S. Malde, J. Rademacker, and G. Wilkinson, Improved determination of the $D \rightarrow$ $K^{-} \pi^{+} \pi^{+} \pi^{-}$coherence factor and associated hadronic parameters from a combination of $e^{+} e^{-} \rightarrow \psi(3770) \rightarrow c \bar{c}$ and $p \bar{p} \rightarrow c \bar{c} \bar{X}$ data, Phys. Lett. B 757, 520 (2016).

[29] Heavy Flavor Averaging Group (HFLAV), http://www.slac .stanford.edu/xorg/hflav/charm/.

[30] S. Malde, C. Thomas, G. Wilkinson, P. Naik, C. Prouve, J. Rademacker, J. Libby, M. Nayak, T. Gershon, and R. A. Briere, First determination of the $C P$ content of $D \rightarrow$ $\pi^{+} \pi^{-} \pi^{+} \pi^{-}$and updated determination of the $C P$ contents of $D \rightarrow \pi^{+} \pi^{-} \pi^{0}$ and $D \rightarrow K^{+} K^{-} \pi^{0}$, Phys. Lett. B 747, 9 (2015). 